\title{
On the Generalized Curvature
}

Tahir H. Ismail

tahir_hsis@uomosul.edu.iq

College of Computer Sciences and Mathematics

University of Mosul

Received on: 18/04/2006
Ibrahim O. Hamad

ibrahim.hamad@su.edu.krd

College of Sciences

University of Salahaddin

Accepted on: 25/06/2006

\section{ABSTRACT}

By using methods of nonstandard analysis given by Robinson, A., and axiomatized by Nelson, E., we try in this paper to establish the generalized curvature of a plane curve $\gamma(t)$ at regular points and at points infinitely close to a singular point. It is known that the radius of curvature of a plane curve $\gamma(t)$ is the limit of the radius of a circle circumscribed to a triangle $\boldsymbol{A B C}$, where $\boldsymbol{B}$ and $\boldsymbol{C}$ are points of $\boldsymbol{\gamma}$ infinitely close to $\boldsymbol{A}$. Our goal is to give a nonstandard proof of this fact. More precisely, if $\boldsymbol{A}$ is a standard point of a standard curve $\gamma$ and $\boldsymbol{B}, \boldsymbol{C}$ are points of $\gamma$ defined by $\boldsymbol{B}=\gamma(\boldsymbol{t}+\boldsymbol{\alpha})$ and $\boldsymbol{C}=\gamma(\boldsymbol{t}+\boldsymbol{\beta})$ where $\alpha$ and $\boldsymbol{\beta}$ are infinitesimals, we intend to calculate the quantity $\frac{\tan \hat{A}}{\|\overrightarrow{B C}\|}$ in the cases where $A$ is biregular,

regular, singular or singular of order $p$.

Keywords: infinitesimals, curvature, torsion, singularity.

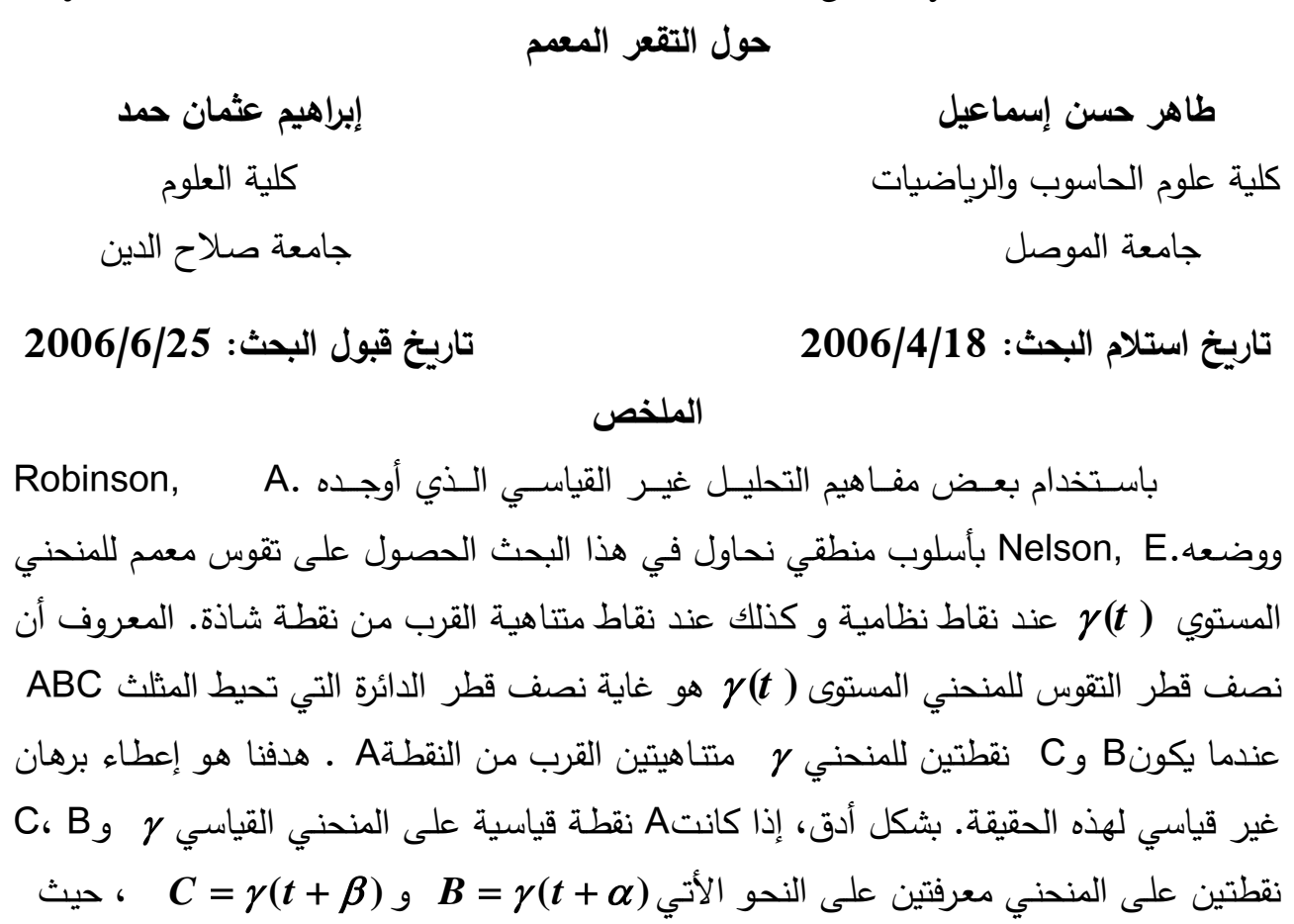




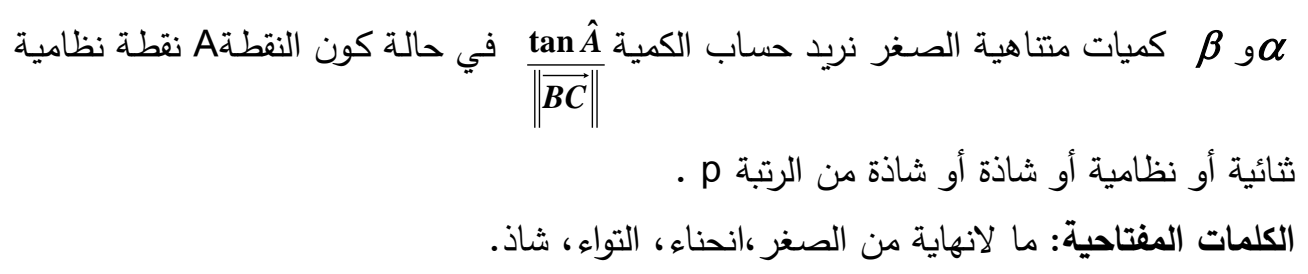

\section{Introduction.}

The radius of curvature is the limit of the radius of a circle circumscribed to triangle $\mathrm{ABC}$, where $\mathrm{B}$ and $\mathrm{C}$ are points of $\gamma$ infinitely close to A [8] [10]. There are many applications of curvature in geometrical study of curves [5], [10] and [26], technology of computer vision and computer graphics [19] and [24], dielectric surfaces [1], chemical interatomic surfaces [17], biological membranes [12], double babbles [18], and other applications.

This study is different from the classical studies of curvature [2], [15] and [19] which deals with the properties of curves in a plane or on a sphere [17], [22]. The following nonstandard definitions are needed throughout this paper.

Every set or elements defined in the classical mathematics are called standard. [11]

Any set or formula, which does not involve new predicates such as "standard, infinitesimals, limited, unlimited...etc", is called internal, otherwise it is called external. [14]

A real number $\boldsymbol{x}$ is called unlimited if and only if $|\boldsymbol{x}|>\boldsymbol{r}$ for all positive standard real numbers $\boldsymbol{r}$, otherwise it is called limited. [4], [3]

The set of all unlimited real numbers is denoted by $\overline{\mathbf{R}}$, and the set of all limited real numbers is denoted by $\underline{\mathbf{R}}$ [23]

A real number $\boldsymbol{x}$ is called infinitesimal if and only if $|\boldsymbol{x}|<\boldsymbol{r}$ for all positive standard real numbers $\boldsymbol{r}$.[9]

A real number $\boldsymbol{x}$ is called appreciable if it is neither unlimited nor infinitesimal, and the set of all positive appreciable numbers is denoted by $\mathrm{A}^{+}$. [25]

Two real numbers $x$ and $y$ are said to be infinitely close if and only if $\boldsymbol{x}-\boldsymbol{y}$ is infinitesimal and denoted by $\boldsymbol{x} \cong \boldsymbol{y}$.[20]

If $\boldsymbol{x}$ is a limited number in $\mathbf{R}$, then it is infinitely close to a unique standard real number, this unique number is called the standard part or shadow of $\boldsymbol{x}$ and it is denoted by $\operatorname{st}(x)$ or ${ }^{0} \boldsymbol{x}$.[21] 
If $f$ is a real valued function then: -

1- $\boldsymbol{f}$ is called continuous at $\boldsymbol{x}_{\boldsymbol{o}}$ if $\boldsymbol{f}$ and $\boldsymbol{x}_{\boldsymbol{o}}$ are standard and if $\boldsymbol{x} \cong \boldsymbol{x}_{\mathbf{0}}$ which implies that $\boldsymbol{f}(\boldsymbol{x}) \cong\left(\boldsymbol{x}_{\mathbf{0}}\right)$ for all $\boldsymbol{x}$.

2- $\boldsymbol{f}$ is called s-continuous at $\boldsymbol{x}_{\mathbf{0}}$ if $\boldsymbol{x} \cong \boldsymbol{x}_{\mathbf{0}}$ which implies that $f(x) \cong\left(x_{0}\right)$ for all $x$.

\section{The Curvature at a Point Near a Study Point}

Today nonstandard analysis rather than it is a branch of mathematics it is a tool used to survey other branches of mathematics and sciences [6], [7], [9], [16].

Meziani [13], Velemorvic [26] and others, studied the curvature via nonstandard analysis. In this paper, the nonstandard tools will be used to present new and more developed forms of curvature.

Let $\boldsymbol{Q}$ and $\boldsymbol{R}$ be two points near to the point $\boldsymbol{P}$ on a curve $\gamma(\boldsymbol{t})$ in a plane as shown in Fig. 2.1. Consider the triangle $\Delta \boldsymbol{Q P R}$ such that points $\boldsymbol{Q}$ and $\boldsymbol{R}$ approach $\boldsymbol{P}$, then the triangle $\Delta \boldsymbol{Q P R}$ is reduced to the triangle $\Delta \boldsymbol{Q} * \boldsymbol{P} \boldsymbol{R} *$. By continuing the approach process of $\boldsymbol{Q} *$ and $\boldsymbol{R} *$ to $\boldsymbol{P}$, we get the infinitesimal triangle $\Delta \boldsymbol{Q} * \boldsymbol{P R} *$ in which $\boldsymbol{Q} *$ and $\boldsymbol{R} *$ become infinitely close to $\boldsymbol{P}$ on $\gamma(\boldsymbol{t})$.

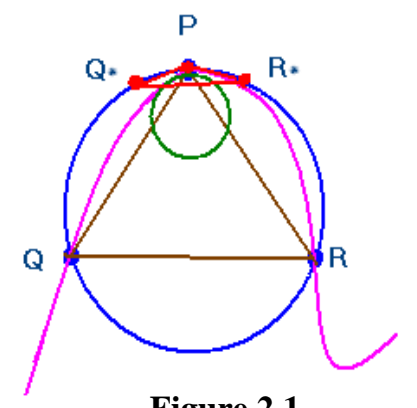

Figure 2.1

\section{Lemma 2.1}

For some $\boldsymbol{t}_{\boldsymbol{o}} \in \mathbf{R}$ let $\boldsymbol{P}=\boldsymbol{\gamma}\left(\boldsymbol{t}_{\boldsymbol{o}}\right)$ be a point on the curve $\boldsymbol{\gamma}$, and let $\boldsymbol{Q} *$ and $\boldsymbol{R} *$ be two points described before, such that $Q_{*}=\gamma\left(t_{o}+\alpha\right)$ and $R *=\gamma\left(t_{o}+\beta\right)$, where $\alpha \cong \mathbf{0}, \beta \cong \mathbf{0}$. Then

1. $Q * \cong P \cong R *$ 
2. The ratio of change of the segment $\overline{Q R}$ with respect to the arc length $\boldsymbol{Q R}$ is infinitely close to the tangent of $\boldsymbol{\gamma}$ at $\boldsymbol{P}$.

3. If the order of $\gamma$ is not less than the order of $\boldsymbol{C}^{\mathbf{2}}$, then any tangent at the point $\boldsymbol{Q} *$ or $\boldsymbol{R} *$ is infinitely close to the tangent of $\boldsymbol{\gamma}$ at $\boldsymbol{P}$, as shown in Fig. 2.1

\section{Proof:}

1. Let $P=\gamma\left(t_{o}\right)=\boldsymbol{x}\left(t_{o}\right) \boldsymbol{e}_{1}+\boldsymbol{y}\left(\boldsymbol{t}_{o}\right) \boldsymbol{e}_{2}$, where $\boldsymbol{e}_{1}, \boldsymbol{e}_{2}$ are basic unit coordinate vectors, then

$$
\begin{aligned}
& Q *=\gamma\left(t_{o}+\alpha\right)=x\left(t_{o}+\alpha\right) e_{1}+y\left(t_{o}+\alpha\right) e_{2} \text { and } \\
& R *=\gamma\left(t_{o}+\beta\right)=x\left(t_{o}+\beta\right) e_{1}+y\left(t_{o}+\beta\right) e_{2} \\
& P-Q_{*}=\left(x\left(t_{o}\right)-x\left(t_{o}+\alpha\right)\right) e_{1}+\left(y\left(t_{o}\right)-y\left(t_{o}+\alpha\right)\right) e_{2} .
\end{aligned}
$$

Since $\gamma$ is a differentiable parametric curve, therefore $\gamma$ is continuous, and $t_{o} \cong t_{o}+\alpha$ so we get $x\left(t_{o}\right) \cong x\left(t_{o}+\alpha\right)$ and $y\left(t_{o}\right) \cong y\left(t_{o}+\alpha\right)$.

Thus $\boldsymbol{Q}_{*} \cong \boldsymbol{P}$, similarly we can prove that $\boldsymbol{P} \cong \boldsymbol{R} *$

2. Let $\overrightarrow{\boldsymbol{T}}\left(\boldsymbol{t}_{\boldsymbol{o}}\right)$ be the tangent vector of $\boldsymbol{\gamma}$ at $\boldsymbol{P}$, then

$$
\vec{T}\left(t_{o}\right)=\left.\frac{d \gamma(t)}{d s}\right|_{t=t_{o}}=\frac{\gamma^{\prime}\left(t_{o}\right)}{\left|\gamma\left(t_{o}\right)\right|} \text {. }
$$

Now $\Delta \gamma=\|\overline{Q R}\|$ and $\Delta s=\|\widehat{Q} R\|$ then as $\Delta s \cong \mathbf{0}$ we get $Q \cong P \cong R$ and conversely.

Thus $\frac{\Delta \gamma}{\Delta s} \cong \frac{d \gamma}{d s}=\frac{\gamma^{\prime}\left(t_{o}\right)}{\left|\gamma\left(t_{o}\right)\right|}=T\left(t_{o}\right)$.

3. Let $\overrightarrow{\boldsymbol{T}}_{\boldsymbol{o}}=\overrightarrow{\boldsymbol{T}}\left(\boldsymbol{t}_{o}\right)$ be the tangent vector of $\boldsymbol{\gamma}$ at $\boldsymbol{P}$, then $T_{o}=\frac{\gamma\left(t_{o}\right)}{\left|\gamma^{\prime}\left(t_{o}\right)\right|}$ and $T_{\alpha}=\left.\frac{d \gamma(t)}{d s}\right|_{t=t_{o}+\alpha}=\frac{\gamma^{\prime}\left(t_{o}+\alpha\right)}{\left|\gamma^{\prime}\left(t_{o}+\alpha\right)\right|}$ since $\gamma^{\prime}$ is continuous, therefore $T_{o} \cong T_{\alpha}$. Similarly we can prove $T_{o} \cong T_{\beta}$, thus $T \alpha \cong T_{o} \cong T_{\beta}$.

\section{3- Generalized Curvature}

The nonstandard principles are used for obtaining a generalized curvature at points infinitely close to regular and singular points. The problem of studying the behavior of curves by curvature at or near singular 
points cannot be obtained with classical differential geometry [8], [15], while with nonstandard analysis this problem can be easily treated [26].

Let $\boldsymbol{A}$ be a standard point on the curve $\boldsymbol{\gamma}$, and let $\boldsymbol{B}$ and $\boldsymbol{C}$ be two points infinitely close to the point $A$, that is $\triangle \boldsymbol{A B C}$ is infinitesimal triangle as shown in Fig. 3.1, then we have the following cases to the point $\boldsymbol{A}$ :

1- $\quad$ If $\gamma^{\prime} \neq \mathbf{0}, \gamma^{\prime \prime} \neq \mathbf{0}$ and $\gamma^{\prime} \cdot \gamma^{\prime \prime} \neq \mathbf{0}$ then the point $\boldsymbol{A}$ is called biregular point.

2- $\quad$ If $\boldsymbol{\gamma}^{\prime} \neq \mathbf{0}$ then the point $\boldsymbol{A}$ is called regular point.

3- $\quad$ If $\gamma^{\prime} \neq \mathbf{0}$ and $\gamma^{\prime} \cdot \gamma^{\prime \prime}=\mathbf{0}$ then the point $\boldsymbol{A}$ is called only regular point.

4- $\quad$ If $\boldsymbol{\gamma}^{\prime}=\mathbf{0}$ then the point is called singular point. In general if $\gamma^{\prime}=\gamma^{\prime \prime}=\cdots=\gamma^{(p-1)}=\gamma^{(p)}=0$ but $\gamma^{(p+1)} \neq \mathbf{0}$ the point $A$ is called singular point of order $p$.

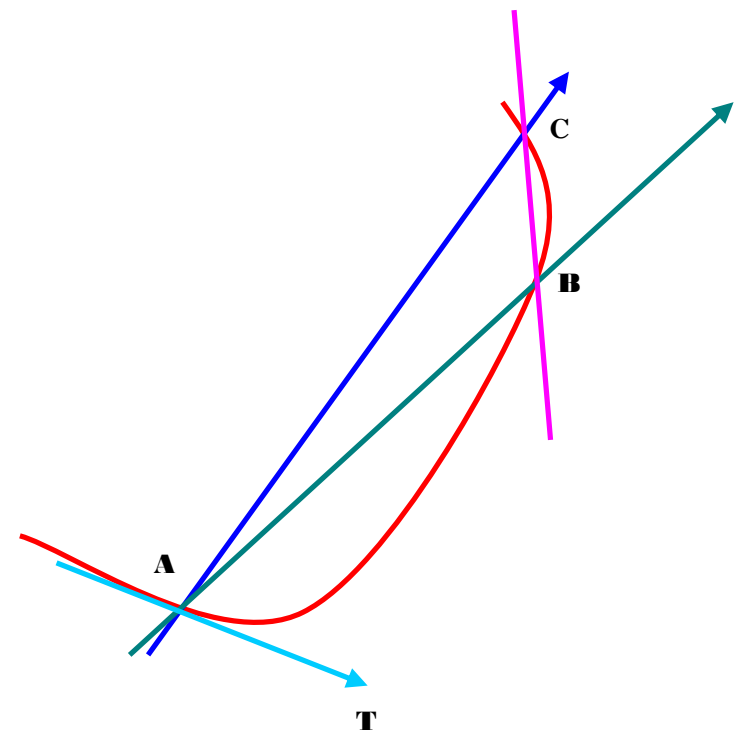

Figure 3.1

\section{Theorem 3.1}

Let $\boldsymbol{A}$ be a standard point on the planar curve $\boldsymbol{\gamma}$, and let $\boldsymbol{B}$ and $\boldsymbol{C}$ be two points infinitely close to the point $\boldsymbol{A}$ as shown in Fig. 3.1, then

1. $\frac{\tan \hat{A}}{\|\overrightarrow{B C}\|}=\frac{\overrightarrow{A C} \times \overrightarrow{A B}}{\frac{1}{2}\|\overrightarrow{B C}\|\left(\|\overrightarrow{A C}\|^{2}+\|\overrightarrow{A B}\|^{2}-\|\overrightarrow{B C}\|^{2}\right)} \cong \frac{\overrightarrow{A C} \times \overrightarrow{A B}}{\|\overrightarrow{B C}\|^{3}} \cong \frac{\overrightarrow{B C} \times \overrightarrow{A B}}{\|\overrightarrow{B C}\|^{3}}$. 
2. $\overrightarrow{B C}=\left.\varepsilon \gamma^{\prime}(t)\right|_{t=t_{o+\alpha}}$
3. $\overrightarrow{A C}=\left.\beta \gamma^{\prime}(t)\right|_{t=t_{o}}$
4. $\overrightarrow{A B}=\left.\alpha \gamma^{\prime}(t)\right|_{t=t_{o}}$

Assuming that the derivatives of orders up to $\boldsymbol{p}$ of $\boldsymbol{\gamma}$ at $\boldsymbol{A}$ exist:

\section{Proof:}

$1-\tan \hat{A}=\frac{\overrightarrow{A C} \times \overrightarrow{A B}}{\overrightarrow{A C} \cdot \overrightarrow{A B}}$ and $\|\overrightarrow{B C}\|^{2}=\|\overrightarrow{A C}\|^{2}+\|\overrightarrow{A B}\|^{2}-2(\overrightarrow{A C} \cdot \overrightarrow{A B})$

Since $\triangle A B C$ is an infinitesimal triangle then the quantities $\|\overrightarrow{\boldsymbol{A C}}\|,\|\overrightarrow{\boldsymbol{A B}}\|$ and $\|\overrightarrow{\boldsymbol{B C}}\|$ are mutually infinitely close. Therefore $\|\overrightarrow{B C}\|^{2}=\overrightarrow{B C} \cdot \overrightarrow{B C} \cong \overrightarrow{A C} \cdot \overrightarrow{A B}$ and

$$
\frac{\tan \hat{A}}{\|\overrightarrow{B C}\|}=\frac{\overrightarrow{A C} \times \overrightarrow{A B}}{\frac{1}{2}\|\overrightarrow{B C}\|\left(\|\overrightarrow{A C}\|^{2}+\|\overrightarrow{A B}\|^{2}-\|\overrightarrow{B C}\|^{2}\right)} \cong \frac{\overrightarrow{A C} \times \overrightarrow{A B}}{\|\overrightarrow{B C}\|^{3}} \cong \frac{\overrightarrow{B C} \times \overrightarrow{A B}}{\|\overrightarrow{B C}\|^{3}}
$$

2- Let $A=\gamma\left(t_{o}\right)=x\left(t_{o}\right) e_{1}+y\left(t_{o}\right) e_{2}$,

$$
\begin{aligned}
& B=\gamma\left(t_{o}+\alpha\right)=x\left(t_{o}+\alpha\right) e_{1}+y\left(t_{o}+\alpha\right) e_{2}, \\
& C=\gamma\left(t_{o}+\beta\right)=x\left(t_{o}+\beta\right) e_{1}+y\left(t_{o}+\beta\right) e_{2}
\end{aligned}
$$

Since $A, B$ and $C$ are not identically equal we may assume $\beta>\boldsymbol{\alpha}$, then $\beta=\alpha+\varepsilon$ for some infinitesimal $\boldsymbol{\varepsilon}$, therefore

$$
\overrightarrow{B C}=\varepsilon\left(\frac{x\left(t_{o}+\alpha+\varepsilon\right)-x\left(t_{o}+\alpha\right)}{\varepsilon}\right) e_{1}+\varepsilon\left(\frac{y\left(t_{o}+\alpha+\varepsilon\right)-y\left(t_{o}+\alpha\right)}{\varepsilon}\right) e_{2}=\left.\varepsilon \gamma^{\prime}(t)\right|_{t=t_{o+\alpha}}
$$

In the same way we can prove the results of 3 and 4 .

\section{Theorem 3.2}

Let $\boldsymbol{A}$ be a standard biregular point on the curve $\boldsymbol{\gamma}$, and let $\boldsymbol{B}$ and $\boldsymbol{C}$ be two points infinitely close to the point $\boldsymbol{A}$, then the usual curvature of the curve $\gamma$ at the point $A$ is given by: $\left(\frac{\tan \hat{A}}{\|\overrightarrow{B C}\|}\right)$

\section{Proof:}


From the previous theorem we have $\overrightarrow{B C}=\left.\varepsilon \gamma^{\prime}(t)\right|_{t=t_{o+\alpha}}$, $\overrightarrow{A C}=\left.\beta \gamma^{\prime}(t)\right|_{t=t_{o}}$ and $\overrightarrow{A B}=\left.\alpha \gamma^{\prime}(t)\right|_{t=t_{o}}$

Since $\gamma$ is continuous

$x\left(t_{o}+\alpha\right)-x\left(t_{o}\right) \cong x\left(t_{o}+2 \alpha\right)-x\left(t_{o}\right) \cong \alpha^{2}\left(\frac{x\left(t_{o}+2 \alpha\right)-2 x\left(t_{o}+\alpha\right)-x\left(t_{o}\right)}{\alpha^{2}}\right)=\alpha^{2} x^{\prime \prime}\left(t_{o}\right) \mathrm{T}$

herefore $\overrightarrow{A B} \cong \alpha^{2} \gamma^{\prime \prime} \Rightarrow \frac{\tan \hat{A}}{\|\overrightarrow{B C}\|}=\frac{\overrightarrow{A C} \times \overrightarrow{A B}}{\|\overrightarrow{B C}\|^{3}}=\frac{\beta \gamma^{\prime} \times \alpha^{2} \gamma^{\prime \prime}}{\left\|\varepsilon \gamma^{\prime}\right\|^{3}} \cong \frac{\gamma^{\prime} \times \gamma^{\prime \prime}}{\left\|\gamma^{\prime}\right\|^{3}}=\kappa$

Thus $\frac{\tan \hat{A}}{\|\overrightarrow{B C}\|} \cong \frac{\gamma^{\prime} \times \gamma^{\prime \prime}}{\left\|\gamma^{\prime}\right\|^{3}}=\kappa$

\section{Remark:}

If $A$ is neither biregular nor regular point then the classical definition of the curvature of $\gamma$ at the point $A$ breaks down, and so the quantity $\frac{\tan \hat{A}}{\|\overrightarrow{B C}\|}$ plays a mean role for determining the behaviors of the curvature vector of $\gamma$ at a point infinitely close to $A$. Also if $A$ is the only regular point of the curve $\gamma$, then the quantity $\tan \hat{A}$ is infinitesimal, but the order of largeness $\overrightarrow{\|\overrightarrow{B C}\|}$

of this infinitesimal with respect to $\|\overrightarrow{B C}\|$ depends on the orders of the first two vector derivatives of $\gamma$ not collinear. Therefore, we can go beyond to the usual region of classical curvature and generalizing the notion of curvature to include all possible cases given at the beginning of this section. For this purpose, we start with the quantity $\frac{\tan \hat{A}}{\|\overrightarrow{B C}\|}$ for defining generalized curvature with respect to the orders of the first two vector derivatives of $\gamma$ not collinear, and denoting it by $\boldsymbol{\kappa}_{G}$.

\section{Theorem 3.3}


Let $\boldsymbol{\gamma}$ be a standard curve of type at least that $\boldsymbol{C}^{2}$, and let $\boldsymbol{A}$ be a standard biregular point on it; and let $\boldsymbol{B}$ and $\boldsymbol{C}$ two points infinitely close to the point $A$. Then $\left(\frac{|\tan \hat{A}|}{\|\overrightarrow{B C}\|}\right) \cong \frac{\left|x^{\prime} y^{\prime \prime}-x^{\prime \prime} y^{\prime}\right|}{2\left(x^{\prime 2}+y^{\prime 2}\right)^{\frac{3}{2}}}=\frac{\left|\gamma^{\prime} \times \gamma^{\prime \prime}\right|}{2 \mid \gamma^{\prime} \|^{3}}$

\section{Proof:}

Let $\quad A=\gamma\left(t_{o}\right)=x\left(t_{o}\right) e_{1}+y\left(t_{o}\right) e_{2}$,

and $\quad \begin{array}{ll}B & =\gamma\left(t_{o}+\alpha\right)=x\left(t_{o}+\alpha\right) e_{1}+y\left(t_{o}+\alpha\right) e_{2}, \\ & =\gamma\left(t_{o}+\beta\right)=x\left(t_{o}+\beta\right) e_{1}+y\left(t_{o}+\beta\right) e_{2}\end{array}$

Expanding each of $\boldsymbol{B}$ and $\boldsymbol{C}$ by Taylor development of the second order, we get

$B=\gamma\left(t_{o}+\alpha\right)=\gamma\left(t_{o}\right)+\alpha \gamma^{\prime}\left(t_{o}\right)+\frac{\alpha^{2}}{2} \gamma^{\prime \prime}\left(t_{o}\right)+\delta_{1} \alpha^{2}$,

and $\quad C=\gamma\left(t_{o}+\beta\right)=\gamma\left(t_{o}\right)+\beta \gamma^{\prime}\left(t_{o}\right)+\frac{\beta^{2}}{2} \gamma^{\prime \prime}\left(t_{o}\right)+\delta_{2} \beta^{2}$.

Then we have

$\|\overrightarrow{B C}\|={ }_{\beta-\alpha} \mid\left[\left(x^{\prime}\left(t_{o}\right)+\frac{\beta+\alpha}{2} x^{\prime \prime}\left(t_{o}\right)+\delta_{3}(\beta-\alpha)\right)^{2}+\left(y^{\prime}\left(t_{o}\right)+\frac{\beta+\alpha}{2} y^{\prime \prime}\left(t_{o}\right)+\delta_{3}(\beta-\alpha)\right)^{2}\right]^{\frac{1}{2}}$,

where $\delta_{2} \beta^{2}-\delta_{1} \alpha^{2}=\delta_{3}(\beta-\alpha)^{2}$. Therefore to find $|\tan \hat{A}|$ we have to find the angular coefficient of each of the lines $\ell_{\mathbf{1}}$ and $\ell_{\mathbf{2}}$ as shown in the Fig.

\section{2.}

The angular coefficient of

$\ell_{1}=\tan (\angle D A B)=\frac{d y / d t}{d x / d t}=\frac{y^{\prime}\left(t_{o}\right)+\frac{\alpha}{2} y^{\prime \prime}\left(t_{o}\right)+\delta_{1} \alpha}{x^{\prime}\left(t_{o}\right)+\frac{\alpha}{2} x^{\prime \prime}\left(t_{o}\right)+\delta_{1} \alpha}$

and the angular coefficient of

$$
\ell_{2}=\tan (\angle D A C)=\frac{d y / d t}{d x / d t}=\frac{y^{\prime}\left(t_{o}\right)+\frac{\beta}{2} y^{\prime \prime}\left(t_{o}\right)+\delta_{2} \beta}{x^{\prime}\left(t_{o}\right)+\frac{\beta}{2} x^{\prime \prime}\left(t_{o}\right)+\delta_{2} \beta}
$$

Then

$$
|\tan (\widehat{A})|=\left|\frac{\tan (\angle D A C)-\tan (\angle D A B)}{1+\tan (\angle D A C) \tan (\angle D A B)}\right|
$$




$$
=\left|\frac{\left(x^{\prime}+\frac{\alpha}{2} x^{\prime \prime}+\delta_{1} \alpha\right)\left(y^{\prime}+\frac{\beta}{2} y^{\prime \prime}+\delta_{1} \beta\right)-\left(x^{\prime}+\frac{\beta}{2} x^{\prime \prime}+\delta_{1} \beta\right)\left(y^{\prime}+\frac{\alpha}{2} y^{\prime \prime}+\delta_{1} \alpha\right)}{\left(x^{\prime}+\frac{\alpha}{2} x^{\prime \prime}+\delta_{1} \alpha\right)\left(y^{\prime}+\frac{\beta}{2} y^{\prime \prime}+\delta_{1} \beta\right)+\left(x^{\prime}+\frac{\beta}{2} x^{\prime \prime}+\delta_{1} \beta\right)\left(y^{\prime}+\frac{\alpha}{2} y^{\prime \prime}+\delta_{1} \alpha\right)}\right|
$$

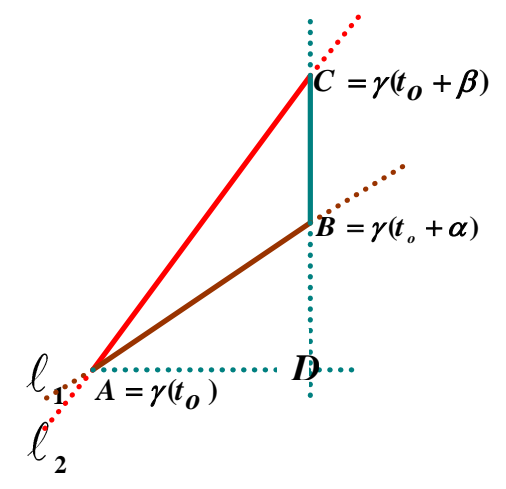

Figure 3.2

Therefore after simplifying the ratio $\frac{|\tan \hat{A}|}{\|\overrightarrow{B C}\|}$ we get: $\frac{|\tan \hat{A}|}{\|\overrightarrow{B C}\|}=\frac{\frac{\beta-\alpha \mid}{2}\left|\left(x^{\prime} y^{\prime \prime}-x^{\prime \prime} y^{\prime}\right)+i . s\right|}{|\beta-\alpha| \mid\left(\left(x^{\prime 2}+i . s\right)+\left(y^{\prime 2}+i s\right)\right)\left(\left(x^{\prime}+i . s\right)^{2}+\left(y^{\prime}+i . s\right)^{2}\right)}$ where i.s represent different infinitesimals. Thus $\frac{|\tan \hat{A}|}{\|\overrightarrow{B C}\|} \cong \frac{\left|x^{\prime} y^{\prime \prime}-x^{\prime \prime} y^{\prime}\right|}{2\left(x^{\prime 2}+y^{\prime 2}\right)^{\frac{3}{2}}}$

From the Theorem 3.3 we deduce that the usual curvature $K(t)$ is infinitely close to twice that of the quantity $\frac{\tan \hat{A}}{\|\overrightarrow{B C}\|}$

The following theorem gives the notion of curvature at a standard only regular point. 


\section{Theorem 3.4}

Let $\gamma$ be a standard curve of order $C^{n}$ and $A$ be a standard only regular point on it, and let $B$ and $C$ be two points infinitely close to the point $A$, then $\left(\frac{|\tan \hat{A}|}{\|\overrightarrow{B C}\|^{q-1}}\right) \cong \frac{\left|x^{\prime} y^{(q)}-x^{(q)} y^{\prime}\right|}{q !\left(x^{\prime 2}+y^{\prime 2}\right)^{q+1}}=\frac{\left|\gamma^{\prime} \times \gamma^{(q)}\right|}{q !\left\|\gamma^{\prime}\right\|^{q+1}}$ where $q$ is the order of the first vector derivative not collinear with $\gamma^{\prime}$

\section{Proof:}

Expand the curve $\gamma$ using Taylor development up to the order $q$ at each of the points $B=\gamma\left(t_{o}+\alpha\right)$ and $C=\gamma\left(t_{o}+\beta\right)$. Then

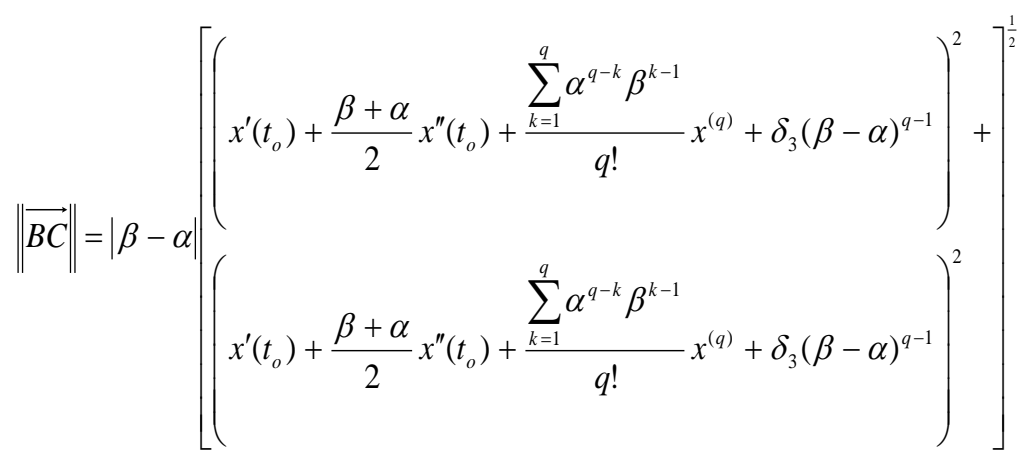

Thus $\|\overrightarrow{B C}\| \cong|\beta-\alpha|\left(x^{\prime 2}+y^{\prime 2}\right)^{\frac{1}{2}}$

Therefore $\|\overrightarrow{B C}\|^{q-1} \cong \mid \beta-\alpha\left(\left(x^{\prime 2}+y^{\prime 2}\right)^{\frac{q-1}{2}}\right.$.

Hence

$$
\begin{aligned}
& \frac{|\tan \hat{A}|}{\|\left.\overrightarrow{B C}\right|^{q-1}}= \\
& \frac{\|\left(\sum_{k=1}^{q} \frac{\alpha^{k-1} x^{(k)}}{k !}+\delta_{1} \alpha^{q-1}\right)\left(\sum_{k=1}^{q} \frac{\beta^{k-1} y^{(k)}}{k !}+\delta_{2} \beta^{q-1}\right)-\left(\sum_{k=1}^{q} \frac{\beta^{k-1} x^{(k)}}{k !}+\delta_{2} \beta^{q-1}\right)\left(\sum_{k=1}^{q} \frac{\alpha^{k-1} y^{(k)}}{k !}+\delta_{1} \alpha^{q-1}\right) \mid}{|\beta-\alpha|\left(\left(\sum_{k=1}^{q} \frac{\alpha^{k-1} x^{(k)}}{k !}+\delta_{1} \alpha^{q-1}\right)\left(\sum_{k=1}^{q} \frac{\beta^{k-1} y^{(k)}}{k !}+\delta_{2} \beta^{q-1}\right)+\left(\sum_{k=1}^{q} \frac{\beta^{k-1} x^{(k)}}{k !}+\delta_{2} \beta^{q-1}\right)\left(\sum_{k=1}^{q} \frac{\alpha^{k-1} y^{(k)}}{k !}+\delta_{1} \alpha^{q-1}\right)\right)\left(x^{12}+y^{2}\right)^{q-1}}
\end{aligned}
$$

Now since $A$ is only regular, then $\boldsymbol{\gamma}^{\prime} \neq \mathbf{0}$; and since the first vector derivative which is not collinear with $\gamma^{\prime}$ is $\gamma^{(q)}$,then all the terms containing 
$\gamma^{\prime} \gamma^{\prime \prime}, \gamma^{\prime} \gamma^{\prime \prime \prime}, \ldots, \gamma^{\prime} \gamma^{(q-1)}$ on the numerator and denometor of the right hand side of equation (3.4.1) vanish.

Therefore $\frac{|\tan \hat{A}|}{\|\overrightarrow{B C}\|^{q-1}} \cong \frac{\left|\left(\frac{\beta}{\alpha}\right)^{q-1}-1\right|\left|x^{\prime} y^{(q)}-x^{(q)} y^{\prime}\right|}{\left|\frac{\beta}{\alpha}-1\right|^{q-1} q !\left(x^{\prime 2}+y^{\prime 2}\right)^{\frac{q+1}{2}}}$.

Since $\alpha$ and $\beta$ are arbitrarily chosen, so we may assume that $\frac{\beta}{\alpha}$ is infinitesimal to get the required result.

\section{Corollary 3.5}

The generalized curvature obtained in the previous theorem is limited and $\left(\frac{|\tan \hat{A}|}{\|\left.\overrightarrow{B C}\right|^{q-1}}\right) \neq 0$.

\section{Proof:}

Expand the curve $\gamma$ using Taylor development up to the order $q$ at each point $B=\gamma\left(t_{o}+\alpha\right)$ and $C=\gamma\left(t_{o}+\beta\right)$, and use the coordinate form of the tangent vector and norm vector to get

$$
\gamma\left(t_{o}+\alpha\right)=\gamma\left(t_{o}\right)+\alpha\left|\gamma^{\prime}\left(t_{o}\right)\right| \vec{T}+\frac{\alpha^{2}}{2} \kappa \vec{N}+O\left(\alpha^{2}\right),
$$

and $\quad \gamma\left(t_{o}+\beta\right)=\gamma\left(t_{o}\right)+\beta\left|\gamma^{\prime}\left(t_{o}\right)\right| \vec{T}+\frac{\beta^{2}}{2} \kappa \vec{N}+O\left(\beta^{2}\right)$ such that

Then using the definition of big $\mathrm{O}$ we find that there exists $\boldsymbol{M} \in \mathbf{N}$

$$
\left|\frac{\gamma\left(t_{o}+\alpha\right)=\gamma\left(t_{o}\right)+\alpha\left|\gamma^{\prime}\left(t_{o}\right)\right| \vec{T}+\frac{\alpha^{2}}{2} \kappa \vec{N}}{\alpha^{2}}\right|<M
$$

That is $\frac{|\mathbf{\kappa}|}{\mathbf{2}}<\boldsymbol{M}$; therefore the curvature $\mathbf{\kappa}$ of $\boldsymbol{\gamma}$ is bounded and so $\frac{|\tan \hat{A}|}{\|\overrightarrow{B C}\|}$ is also bounded. Now we prove that $\frac{|\tan \hat{A}|}{\|\overrightarrow{B C}\|^{q-1}}$ is limited. 
Since $\frac{|\tan \hat{A}|}{\|\overrightarrow{B C}\|^{q-1}}=\frac{|\tan \hat{A}|}{\|\overrightarrow{B C}\| \overrightarrow{B C} \|^{q-2}}=\frac{\kappa}{\|\overrightarrow{B C}\|^{q-2}}$, then we have the following two cases:

If $\|\overrightarrow{B C}\|^{\boldsymbol{q}-2}$ is not infinitesimal, then the result is obtained at once.

If $\|\overrightarrow{\boldsymbol{B C}}\|^{\boldsymbol{q}-\mathbf{2}}$ is infinitesimal, use Archimedean property to get that $\frac{\kappa}{\|\overrightarrow{B C}\|^{q-2}}$ is limited.

The proof of $\left(\frac{|\tan \hat{A}|}{\|\overrightarrow{B C}\|^{q-1}}\right) \neq \mathbf{0}$ is by contradiction. Since the only standard infinitesimal is zero, then $\frac{|\tan \hat{A}|}{\|\overrightarrow{B C}\|^{q-1}}=\mathbf{0}$ or $\frac{|\tan \hat{A}|}{\|\overrightarrow{B C}\|^{q-1}} \cong \mathbf{0}$.

Thus either ${ }^{o}(\tan \hat{A})=\mathbf{0}$ or $\|\overrightarrow{B C}\|$ is unlimited. In the first case, we get that $\overrightarrow{\boldsymbol{A B}}$ and $\overrightarrow{\boldsymbol{A C}}$ are collinear which is a contradiction. The second case is impossible.

\section{Theorem 3.6}

Let $\gamma$ be a standard curve of order $\boldsymbol{C}^{\boldsymbol{n}}$ and A be a standard singular point of order $p-1$ on $\gamma$; and let $B$ and $C$ be two points internally close to the point $A$, then

$$
\left(\frac{|\tan \hat{A}|}{\|\overrightarrow{B C}\|^{\frac{q}{p}-1}}\right)=\frac{(p !)^{\frac{q}{p}}\left|x^{(p)} y^{(q)}-x^{(q)} y^{(p)}\right|}{q !\left(x^{(p)^{2}}+y^{(p)^{2}}\right)^{\frac{q+p}{2 p}}}=\frac{(p !)^{\frac{q}{p}}\left|\gamma^{(p)} \times \gamma^{(q)}\right|}{q !\left\|\gamma^{(p)}\right\|^{\frac{q}{p}+1}} \text {, where } q \text { is the order }
$$

of the first vector derivative of $\gamma$ not collinear with $\gamma^{(p)}$.

\section{Proof:}


Expanding the curve $\boldsymbol{\gamma}$ using Taylor development up to the order $\boldsymbol{q}$ at points $B=\gamma\left(t_{o}+\alpha\right)$ and $C=\gamma\left(t_{o}+\beta\right)$, we get

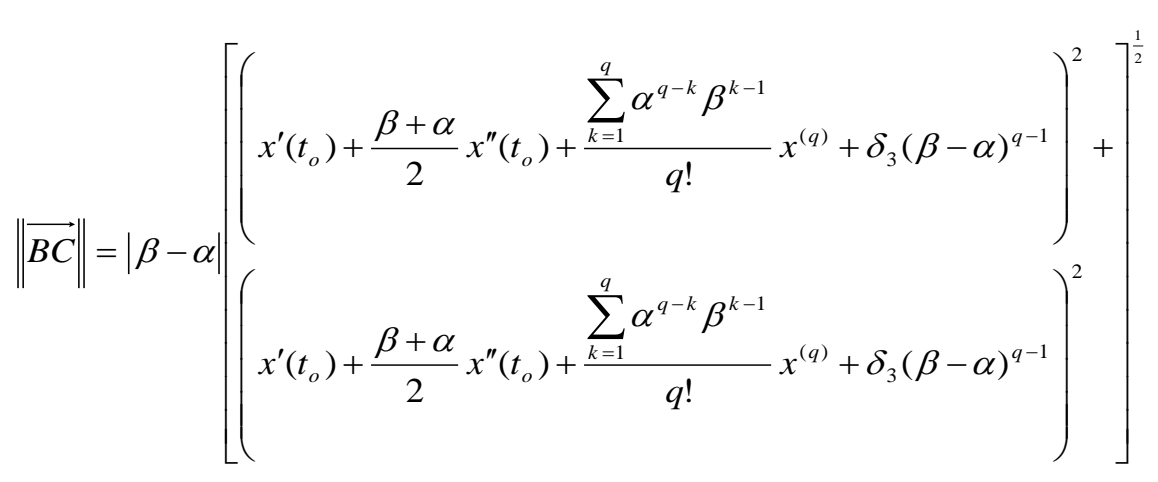

Since $\boldsymbol{A}$ is a singular point of order $\boldsymbol{p}-\boldsymbol{1}$ we get $\gamma^{\prime}=\gamma^{\prime \prime}=\cdots=\gamma^{(p-1)}=\mathbf{0}$ and $\gamma^{(p)} \neq \mathbf{0}$ then $\|\overrightarrow{B C}\| \cong \frac{\left|\beta^{p}-\alpha^{p}\right|}{p !}\left(x^{(p)^{2}}+y^{(p)^{2}}\right)^{\frac{1}{2}}$

Therefore $\|\overrightarrow{B C}\|^{\frac{q}{p}-1} \cong \frac{\left|\beta^{p}-\alpha^{p}\right|^{\frac{q}{p}-1}}{p !}\left(x^{\prime 2}+y^{\prime 2}\right)^{\frac{q-p}{2 p}}$.

Thus

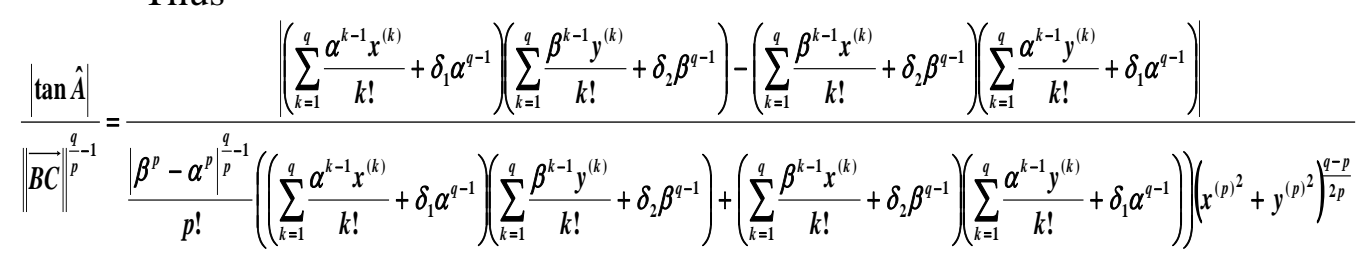

Therefore 


$$
\frac{|\tan \hat{A}|}{\|\overrightarrow{B C}\|^{\frac{q}{p}-1}}=\frac{\left(\frac{\alpha^{p-1} \beta^{q-1} x^{(p)} y^{(q)}}{p ! q !}+\frac{\alpha^{q-1} \beta^{p-1} x^{(q)} y^{(p)}}{p ! q !}+i . s\right)-\left(\frac{\alpha^{q-1} \beta^{p-1} x^{(p)} y^{(q)}}{p ! q !}+\frac{\alpha^{p-1} \beta^{q-1} x^{(q)} y^{(p)}}{p ! q !}+i . s\right)}{\frac{\left|\beta^{p}-\alpha^{p}\right|^{\frac{q}{p}-1}}{p !}\left(\left(\frac{\alpha^{p-1} \beta^{q-1}}{p ! p !} x^{(p)} x^{(p)}+i . s\right)+\left(\frac{\alpha^{p-1} \beta^{q-1}}{p ! p !} y^{(p)} y^{(p)}+i . s\right)+\right)\left(x^{(p)^{2}}+y^{(p)^{2}}\right)^{q-p}}
$$

$$
\left(\frac{|\tan \hat{A}|}{\|\overrightarrow{B C}\|^{\frac{q}{p}-1}}\right)=\frac{(p !)^{q}}{q !} \cdot\left|\frac{\left(\frac{\beta}{\alpha}\right)^{q-1}-1}{\left(\left(\frac{\beta}{\alpha}\right)^{p}-1\right)^{\frac{q}{p}-1}}\right| \frac{(p !)^{\frac{q}{p}}\left|x^{(p)} y^{(q)}-x^{(q)} y^{(p)}\right|}{q !\left(x^{(p)^{2}}+y^{(p)^{2}}\right)^{q+p}} .
$$

Since $\alpha$ and $\beta$ are arbitrarily chosen, so we may assume that $\frac{\beta}{\alpha}$ is infinitesimal to get the required result.

Now, since $\boldsymbol{A}$ is only regular, then $\boldsymbol{\gamma}^{\prime} \neq \mathbf{0}$ and since the first vector derivative which is not collinear with $\gamma^{\prime}$ is $\gamma^{(q)}$, then we find that all the terms containing $\gamma^{\prime} \gamma^{\prime \prime}, \gamma^{\prime} \gamma^{\prime \prime \prime}, \ldots, \gamma^{\prime} \gamma^{(\boldsymbol{q}-1)}$ on the numerator and denometor of the right hand side of equation (3.6.1) vanish.

Therefore $\frac{|\tan \hat{A}|}{\|\overrightarrow{B C}\|^{q-1}}=\frac{\left|\left(\frac{\beta}{\alpha}\right)^{q-1}-1\right|\left|x^{\prime} y^{(q)}-x^{(q)} y^{\prime}\right|}{\left|\frac{\beta}{\alpha}-1\right|^{q-1} q !\left(x^{\prime 2}+y^{\prime 2}\right)^{\frac{q+1}{2}}}$.

Similarly, from equation $($ 3.6.2), we get:

$$
\left(\frac{|\tan \hat{A}|}{\|\overrightarrow{B C}\|^{\frac{q}{p}-1}}\right)=\frac{(p !)^{\frac{q}{p}}\left|x^{(p)} y^{(q)}-x^{(q)} y^{(p)}\right|}{q !\left(x^{(p)^{2}}+y^{(p)^{2}}\right)^{q+p}}=\frac{(p !)^{\frac{q}{p}}\left|\gamma^{(p)} \times \gamma^{(q)}\right|}{q !\left\|\gamma^{(p)}\right\|^{\frac{q}{p}+1}} .
$$




\section{REFERENCES}

[1] Antoine, C. G. and G. Soriano, (2004) "Weighted curvature approximation- numerical tests for 2D dielectric surfaces", Waves Random Media, Vol. 14, pp. 349- 363.

[2] Bandos, I. A. and J. A. de Azcdrraga, (2005) "Generalized curvature and the equations of $\mathrm{D}=11$ ", IFIC/04-74, FTUV-05-0101 [arXiv: hepth/ 0501007 v2].

[3] Davis, M. (1977) Applied Nonstandard Analysis, New York: Wiley.

[4] Diener, F.and Diener M. (1996) Nonstandard Analysis in Practice, Springer-Verlag, Berlin,HeildeBerg.

[5] Dyn,N. and K. rmann, (2000) "Optimizing 3D Triangulations Using Discrete Curvature Analysis Mathematical Methods for Curves and Surfaces", Tom Lyche and Larry L. Schumaker (eds.), Oslo, pp.135-146.

[6] Fleuriot, J. D. (2001) "Theorem Proving in Infinitesimal Geometry", Division of Informatics, University of Edinburgh, J of the IGPL, Vol.9, No3, pp.447-474.

[7] Giordano, P. (2004) "Infinitesimal Differential Geometry", Acta Math Univ. Com. Vol. LXXIII, No.2 ,pp. 235- 278.

[8] Gudmundsson, S. (2004) An Introduction to Riemannian Geometry, Lecture Notes version 1.235 - 9 December.

[9] Keisler, H. J. (2005) Elementary Calculus-2 ${ }^{\text {ed-An Infinitesimal }}$ Approach, Creative Commons, 559 Nathan Abbott, Stanford, California, 93405, USA.

[10] Koláŕ, I. and P. W. Slovák, (1993) Natural Operations in Differential Geometry, Springer-Verlag, Berlin Heidelberg.

[11] Lutz R. and M. Goze (1982) Nonstandard Analysis -A Practical Guide with Application, Lecture Notes in math. (881), Springier, Berlin.

[12] Marques, C.M and J. B. Fournier, (1996) "Deviatoric Spontaneous curvature of lipid membranes induced by Siamese macromolecular co surfactants", Europhys. Lett. , Vol. 35, No. 5, pp.361-365.

[13] Meziani, A. (2003) "Infinitesimal Bendings of Homogeneous Surfaces with Nonnegative Curvature", Comm. Anal. Geom., Vol.11, No.4 pp.697-719. 
[14] Nelson, E. (1977) "Internal set Theory - A New Approach to Nonstandard Analysis", Bull. Amer. Math. Soc, Vol.83, No.6, pp.1165-1198.

[15] Nishimura, H. (2000) "Another Curvature in Synthetic Differential Geometry”, Bull. Belg. Math. Soc. Vol.7, pp.161-171.

[16] Păsărescu, A. (2004) "On Some Applications of Nonstandard Analysis in Geometry", Differential Geometr-Dynamical Systems, Vol. 16, pp.23- 30.

[17] Pendas M. A. and V. Luana (2003) "Curvature of Interatomic Surfaces I.", Fundamentals Journal of Chemical Physics Vol. 119, No. 15, pp.7633-7643.

[18] Reichardt, B. W. and C. Heilmann, (2003) "Proof of the Double Bubble Conjecture in $\mathrm{R}^{4}$ and Certain Higher Dimensional Cases", Pacific Journal of Mathematics, Vol. 208, No. 2, pp. 347- 366.

[19] Rieger, B. and J. Frederik, (2004) "On Curvature Estimation of Surfaces in 3D Gray- Value Images and the Computation of Shape Descriptors", IEEE Transactions on Image Processing, Vol. 26, No. 8.

[20] Robinson, A. (1974) Nonstandard Analysis-2 ${ }^{\text {ED }}$, North-Holland Pub. Com.

[21] Rosinger, E. E (2004) "Short Introduction to Nonstandard Analysis", arXiv: math. GM/ 0407178 v1 $10 \mathrm{Jul}$.

[22] Steiner, D. C. and H. Edelsbrunner (2005) "Inequalities for the Curvature of Curves and Surfaces", SCG'05, June 6- 8, Pisa, Italy.

[23] Stroyan, K. D. and W. A. Luxemburg, (1976) Introduction to the Theory of Infinitesimals, New York, Academic Press.

[24] Utcke, S. (2003) "Error Boundson Curvature Estimation", L.D Giffin and M. Lillholm(Eds)Scale-Space LNCS, pp.657-666.

[25] Van Den Berg (1984) "I. Halos and Galaxies: Application on the Real Line", Serie de math. Pures et Appliquees, IRMAStrasbourg.

[26] Velimirovic, L. S. and S. M. Mincic (2002) "Infinitesimal Deformations of Curvature Tensors at Non Symmetric Affine Connection Space", МАТЕМАИЧК ВЕСНИК 54, pp.219226. 